\title{
Contribution of obstructive sleep apnoea to arterial stiffness: a meta-analysis using individual patient data
}

\author{
Marie Joyeux-Faure, ${ }^{1,2}$ Renaud Tamisier, ${ }_{1}^{1,2}$ Jean-Christian Borel, ${ }_{1,2}^{1,2}$ \\ Sandrine Millasseau, ${ }^{3}$ Louis-Marie Galerneau, ${ }^{1,2}$ Marie Destors, ${ }^{1,2}$ Sébastien Bailly, ${ }^{1,2}$ \\ Jean Louis Pepin ${ }^{1,2}$
}

\begin{abstract}
- Additional material is published online only. To view please visit the journal online (http://dx.doi.org/10.1136/ thoraxjn-2018-211513).

${ }^{1}$ Laboratoire HP2, INSERM U1042, Univ. Grenoble Alpes, Grenoble, France 'Laboratoire EFCR, Grenoble Alpes University Hospital, Grenoble, France

${ }^{3}$ Pulse Wave Consulting, Saint Leu la Foret, France
\end{abstract}

\section{Correspondence to}

Professor Jean Louis Pepin, EFCR laboratory, Grenoble Alpes University Hospital, Grenoble CP10217 38043, France; jpepin@chu-grenoble.fr

SB and JLP contributed equally.

Received 10 January 2018 Revised 8 July 2018

Accepted 9 July 2018

Published Online First

21 July 2018

\section{GLinked}

- http://dx.doi.org/10.1136/ thoraxjnl-2018-212332

Check for updates

(c) Author(s) (or their employer(s)) 2018. No commercial re-use. See rights and permissions. Published by BMJ.

To cite: Joyeux-Faure $\mathrm{M}_{\text {, }}$ Tamisier R, Borel J-C, et al.

Thorax 2018;73:1146-1151.

\section{ABSTRACT}

Background Arterial stiffness, measured by pulse wave velocity (PWV), is a strong independent predictor of late cardiovascular events and mortality. It is recognised that obstructive sleep apnoea (OSA) is associated with cardiovascular comorbidities and mortality. Although previous meta-analyses concluded that PWV is elevated in OSA, we feel that an individual patient data analysis from nine relatively homogeneous studies could help answer: to what extent does OSA drive arterial stiffness? Methods Individual data from well-characterised patients referred for suspicion of OSA, included in nine studies in which carotid-femoral PWV was measured using a Complior device, were merged for an individual patient data meta-analysis.

Results 893 subjects were included (age: $56 \pm 11$ (mean \pm SD), $72 \%$ men, 84\% with confirmed OSA). Body Mass Index varied from 15 to $81 \mathrm{~kg} / \mathrm{m}^{2}(30 \pm 7 \mathrm{~kg} /$ $\left.\mathrm{m}^{2}\right)$. PWV ranged from 5.3 to $20.5 \mathrm{~m} / \mathrm{s}(10.4 \pm 2.3 \mathrm{~m} / \mathrm{s})$. In univariate analysis, $\log (\mathrm{PWV})$ was strongly related to age, gender, systolic blood pressure, presence of type 2 diabetes (all $p<0.01$ ) as well as to dyslipidaemia $(\mathrm{p}=0.03)$ and an Epworth Sleepiness Scale score $\geq 9(p=0.04)$, whereas it was not related to obesity $(p=0.54)$, a severe Apnoea-Hypopnoea Index $(p=0.14)$, mean nocturnal saturation $(p=0.33)$ or sleep time with oxygen saturation below $90 \%(p=0.47)$. In multivariable analysis, PWV was independently associated with age, systolic blood pressure and diabetes (all $p<0.01$ ), whereas severe OSA was not significantly associated with PWV.

Conclusion Our individual patient meta-analysis showed that elevated arterial stiffness in patients with OSA is driven by conventional cardiovascular risk factors rather than apnoea parameters.

\section{INTRODUCTION}

Arteries carry oxygen and nutriments and also attenuate cardiac pulsatility due to their elastic properties. With ageing and in the presence of cardiovascular disease, large arteries, in particular the aorta, tend to stiffen. ${ }^{1}$ In the European Hypertension and Cardiology Society guidelines, elevated arterial stiffness is recognised as a strong predictor of end-organ damage and cardiovascular mortality that is independent of other usual cardiovascular risk factors. ${ }^{2-4}$ The international expert committee recommends carotid-femoral pulse wave velocity

\section{Key messages}

What is the key question?

- What are the relative impacts of obstructive sleep apnoea severity and conventional risk factors on arterial stiffness?

What is the bottom line?

- The elevation of arterial stiffness in patients with obstructive sleep apnoea is driven more by the conventional cardiovascular risk factors (age, hypertension and the presence of diabetes) than by apnoea severity indices.

Why read on?

- This meta-analysis used individual patient data from nine studies performed by the same centre with 893 well-characterised patients and pulse wave velocity measured using the same technique for all studies.

(PWV) as the 'gold standard' for the assessment of aortic stiffness. ${ }^{1}$ Patients with chronic diseases that have high cardiovascular risk such as hypertension or diabetes exhibit higher PWV. ${ }^{5}$ However, contrary to what may be intuitively expected, in obese subjects, conflicting results regarding arterial stiffness are reported. This might be partly explained by heterogeneity of obesity patients including its association, or not, with obstructive sleep apnoea (OSA).

OSA syndrome is strongly associated with cardiovascular morbidity and mortality. ${ }^{8}$ Data summarised in a systematic review by Doonan $e t a l^{9}$ and later in a critical review by Phillips et $a l^{10}$ reported that patients with severe OSA have elevated PWV compared with healthy volunteers or control groups exhibiting no or mild OSA. ${ }^{11-17}$ A relationship between PWV and OSA severity ${ }^{11} 18$ was suggested but was not systematically confirmed. ${ }^{14} 1920$

OSA syndrome is associated with several clusters of comorbidities $^{21}$ such as hypertension, ${ }^{22}$ obesity, ${ }^{23}{ }^{24}$ diabetes, coronary heart disease and cardiac failure. These coexisting chronic diseases also each strongly contribute towards elevated PWV. ${ }^{5}$ The relationship between OSA severity and arterial stiffness assessed by PWV needs clarifying particularly as previous meta-analyses and reviews 
in the field have included heterogeneous studies with relatively limited sample sizes and poorly described comorbidities. ${ }^{1025}$ To determine the relative impacts of OSA severity and conventional risk factors on arterial stiffness, we conducted an individual patient data meta-analysis on data from a large group of nearly 900 patients with suspicion of OSA, explored in the same centre, for whom PWV and comorbidities were systematically and carefully assessed.

\section{METHODS}

Individual data from participants involved in separate studies performed by our group between 2006 and 2015 were pooled. We excluded studies in which PWV was recorded for less than $80 \%$ of patients, or in which there was heterogeneity in the PWV or polysomnography (PSG) methods used. Only data from baseline measurements from observational studies and randomised clinical trials were used in this individual data meta-analysis.

All studies were conducted in accordance with applicable good clinical practice requirements in Europe, French law, ICH E6 recommendations and the ethical principles of the Helsinki Declaration (1996 and 2000). They were approved by an independent Ethics Committee and registered on the ClinicalTrials. gov site (online supplementary table S1).

\section{Clinical evaluation}

Hypertension was defined as systolic blood pressure (SBP) greater than $140 \mathrm{~mm} \mathrm{Hg}$, diastolic blood pressure greater than $90 \mathrm{~mm} \mathrm{Hg}$ or need for antihypertensive treatment. Clinical blood pressure (BP) was measured in triplicate using a mercury sphygmomanometer or with validated oscillometric devices. The average of three measurements was used. Diabetes was defined based on current use of antidiabetic medications and dyslipidaemia on current lipid-lowering medications. Body Mass Index (BMI) was determined and obesity classes were defined according to WHO definitions: normal, BMI $<25 \mathrm{~kg} / \mathrm{m}^{2}$; overweight, $25 \leq \mathrm{BMI}<30 \mathrm{~kg} / \mathrm{m}^{2}$; obese, $30 \leq \mathrm{BMI}<35 \mathrm{~kg} / \mathrm{m}^{2}$; severely obese, $35 \leq \mathrm{BMI}<40 \mathrm{~kg} / \mathrm{m}^{2}$; and morbidly obese, $\geq 40 \mathrm{~kg} / \mathrm{m}^{2}$.

\section{Sleep studies}

All patients were diagnosed with OSA using overnight respiratory polygraphy or PSG, performed with spontaneous breathing to characterise abnormal respiratory events during sleep. Details of the procedure as well as of the definitions of apnoea and hypopnoea can be found in the e-Appendix 1 of our previous publication. ${ }^{26}$ The Apnoea-Hypopnoea Index (AHI) was calculated as the number of apnoea and hypopnoea events per hour of sleep (full PSG). Patients also completed the Epworth Sleepiness Scale (ESS). Subjects were classified as controls if AHI $<5$ events/hour, or suffering from mild $(5 \leq \mathrm{AHI}<15$ events/hour), moderate $(15 \leq \mathrm{AHI}<30$ events/hour) or severe OSA (AHI $\geq 30$ events/ hour). Mean nocturnal oxygen saturation $\left(\mathrm{SaO}_{2}\right)$ and time spent under $90 \%$ of $\mathrm{SaO}_{2}$ were also collected in order to characterise sleep apnoea severity. Overnight sleep studies were scored according to international guidelines. ${ }^{27}$

\section{Arterial stiffness assessed by PWV}

PWV was measured using the same technique for all studies: carotid and femoral waveforms were acquired with the Complior SP device (Alam Medical, France). The validation and reproducibility of Complior measurements have been published elsewhere. ${ }^{2829}$ PWV is automatically calculated by the Complior from the transit time between simultaneous carotid and femoral tracings and the arterial distance between these two sites. At the time of the studies, the arterial distance pathway was estimated from surface measurements and later multiplied by 0.8 as advised by the latest expert consensus on PWV measurements. ${ }^{30}$ At least two measurements of PWV were systematically performed. When the difference between measurements was less than $0.5 \mathrm{~m} / \mathrm{s}$, the mean value was kept. When the difference was above $0.5 \mathrm{~m} / \mathrm{s}$, a third measurement was done and the median value was taken into account. The same procedure and methodology was conducted across studies.

\section{Statistical analysis}

Qualitative variables were described using numbers and proportion and quantitative variables are described using

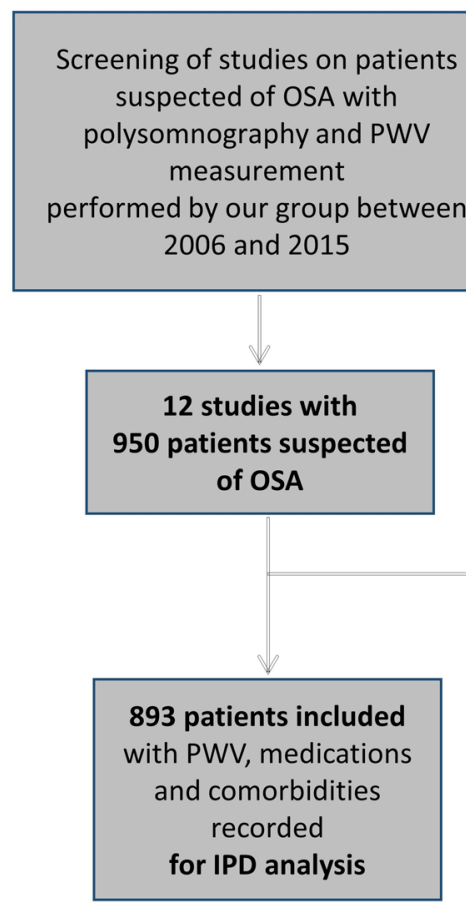

Figure 1 Flowchart of selected studies and patients. OSA, obstructive sleep apnoea; PWV, pulse wave velocity. 
Table 1 Patients' characteristics for the whole population

\begin{tabular}{|c|c|}
\hline & $\mathrm{n}=893$ \\
\hline Male $(\mathrm{n}(\%))$ & $644(72)$ \\
\hline Age (years) & $56 \pm 11$ \\
\hline BMI $\left(\mathrm{kg} / \mathrm{m}^{2}\right)$ & $29.9 \pm 6.9$ \\
\hline Normal (n (\%)) & $199(22)$ \\
\hline Overweight (n (\%)) & $345(39)$ \\
\hline Obese (n (\%)) & $163(18)$ \\
\hline Severely obese (n (\%)) & $106(12)$ \\
\hline Morbidly obese (n (\%)) & $80(9)$ \\
\hline Hypertension (n (\%)) & $694(78)$ \\
\hline Type 2 diabetes ( $\mathrm{n}(\%))$ & $401(45)$ \\
\hline Dyslipidaemia (n (\%)) & $551(62)$ \\
\hline OSA (n (\%)) & $683(84)$ \\
\hline $\mathrm{SBP}(\mathrm{mm} \mathrm{Hg})$ & $133 \pm 17$ \\
\hline $\mathrm{DBP}(\mathrm{mm} \mathrm{Hg})$ & $81 \pm 12$ \\
\hline $\mathrm{HR}(\mathrm{bpm})$ & $69 \pm 12$ \\
\hline $\mathrm{PWV}(\mathrm{m} / \mathrm{s})$ & $10.4 \pm 2.3$ \\
\hline AHI (events/h) & $36.8 \pm 24.1$ \\
\hline Mean nocturnal $\mathrm{SaO}_{2}(\%)$ & $92.9 \pm 2.7$ \\
\hline $\mathrm{SaO}_{2}<90 \%$ ( $\%$ of recording time) & $10.8 \pm 18.3$ \\
\hline ESS score & $9.5 \pm 5.2$ \\
\hline
\end{tabular}

Results are observed data. They are expressed as mean \pm SD or $n(\%)$. These percentages are before imputation of missing data. The imputation of missing data is shown in online supplementary table $\mathrm{S3}$.

AHI, Apnoea-Hypopnoea Index; BMI, Body Mass Index; DBP, diastolic blood pressure; ESS, Epworth Sleepiness Scale; HR, heart rate; OSA, obstructive sleep apnoea; $\mathrm{PWV}$, pulse wave velocity; $\mathrm{SaO}_{2}$, oxygen saturation; $\mathrm{SaO}_{2}<90 \%$, percentage of recording time spent at a $\mathrm{SaO}_{2}<90 \%$; $\mathrm{SBP}$, systolic blood pressure.

mean and SD. Missing values were imputed using a multiple imputation method when the proportion of missing values was $<20 \%$. Five datasets were generated using logistic regression for qualitative variables or linear regression for quantitative variables, considering that missing values were random. More information about data imputation methods are given in online supplementary table S3. As PWV distribution was skewed, the analysis was first performed with $\log (\mathrm{PWV})$. However, a sensitivity analysis using untransformed PWV showed that the results did not differ from those using $\log (\mathrm{PWV})$. Hence, we re-analysed and present results from non-log-transformed PWV for easier interpretation by clinicians and readers.

A one-step individual patient data (IPD) meta-analysis was performed to adjust on patient variables. A univariate linear mixed model with a random effect by study was built to identify factors associated with PWV. Factors significantly associated with PWV and severe OSA (AHI $\geq 30$ events/hour) were introduced in a multivariate linear mixed model to identify independent factors associated with PWV. A backward selection was performed to identify the final multivariable model.

Comparisons of patients' characteristics according to the AHI were performed using a univariate linear mixed model for quantitative values or logistic regression for qualitative ones.

$P$ values $<0.05$ were considered statistically significant. Data were analysed using SAS V.9.4 (SAS Institute, Cary, North Carolina, USA).

\begin{tabular}{|c|c|c|c|c|}
\hline & $\begin{array}{l}\text { Controls } \\
\mathrm{AHI}<5\end{array}$ & $\begin{array}{l}\text { Mild } \\
5 \leq A H I<15\end{array}$ & $\begin{array}{l}\text { Moderate } \\
15 \leq A H I<30\end{array}$ & $\begin{array}{l}\text { Severe } \\
\mathrm{AHI} \geq 30\end{array}$ \\
\hline $\mathrm{N}$ & 58 & 97 & 237 & 501 \\
\hline Male n (\%) & $30(52)$ & $55(57)$ & $168(71)$ & $392(78)^{*}$ \\
\hline Age (years) & $52.0 \pm 13.1$ & $56.4 \pm 13.6^{*}$ & $57 \pm 11.3^{*}$ & $56.0 \pm 11.5^{*}$ \\
\hline BMI $\left(\mathrm{kg} / \mathrm{m}^{2}\right)$ & $29.3 \pm 7.9$ & $29 \pm 7.5$ & $28.7 \pm 6.8$ & $30.7 \pm 6.9$ \\
\hline Hypertension (n (\%)) & $33(56)$ & $65(67)$ & $185(78)$ & $413(82)^{*}$ \\
\hline Type 2 diabetes ( $\mathrm{n}(\%))$ & $19(33)$ & $48(50)$ & $106(45)$ & $227(45 \%)$ \\
\hline Dyslipidaemia (n (\%)) & $34(59)$ & $56(58)$ & $138(58)$ & $323(64)$ \\
\hline $\mathrm{SBP}(\mathrm{mm} \mathrm{Hg})$ & $126 \pm 18$ & $131 \pm 18$ & $134 \pm 19^{*}$ & $134 \pm 17^{*}$ \\
\hline $\mathrm{DBP}(\mathrm{mm} \mathrm{Hg})$ & $74 \pm 15$ & $74 \pm 12$ & $81 \pm 13^{*}$ & $82 \pm 12^{*}$ \\
\hline HR (bpm) & $68 \pm 15$ & $67 \pm 14$ & $69 \pm 13$ & $71 \pm 15$ \\
\hline $\mathrm{PWV}(\mathrm{m} / \mathrm{s})$ & $10.1 \pm 2.6$ & $10.7 \pm 3$ & $10.3 \pm 2.4$ & $10.4 \pm 2.3$ \\
\hline $\begin{array}{l}\text { Mean nocturnal } \\
\mathrm{SaO}_{2}(\%)\end{array}$ & $93.6 \pm 2.3$ & $93.2 \pm 2.5$ & $93.1 \pm 3.1$ & $92.6 \pm 2.9^{*}$ \\
\hline $\mathrm{SaO}_{2}<90 \%(\%)$ & $6.1 \pm 16.6$ & $6.8 \pm 14.3$ & $6.4 \pm 14,1$ & $14.0 \pm 20.9^{*}$ \\
\hline ESS score & $7.9 \pm 5.7$ & $8.5 \pm 8.4$ & $9.2 \pm 6.5$ & $10 \pm 5.3^{*}$ \\
\hline
\end{tabular}

Results are observed data. They are expressed as mean \pm SD or $n(\%)$. These percentages are before imputation of missing data.

* $P$ value versus controls $<0.01$.

AHI, Apnoea-Hypopnoea Index (events/hour); BMI, Body Mass Index; DBP, diastolic blood pressure; ESS, Epworth Sleepiness Scale; HR, heart rate; PWV, pulse wave velocity; $\mathrm{SaO}_{2}$, oxygen saturation; $\mathrm{SaO}_{2}<90 \%$, percentage of recording time spent at a $\mathrm{SaO}_{2}<90 \%$; $\mathrm{SBP}$, systolic blood pressure.

\section{RESULTS}

The nine studies included in the meta-analysis are detailed in the online supplement in terms of regulatory approval, design and publications (figure 1 and online supplementary table S1). All these studies concerned patients with OSA or suspected of OSA. There were six randomised and three observational studies.

The characteristics of the whole group of 893 patients are shown in table 1 and patients' characteristics per study are shown in online supplementary table S2. Most subjects were men (72\%) and $84 \%$ had confirmed OSA syndrome. We had a large range of PWV: from 5.3 to $20.5 \mathrm{~m} / \mathrm{s}$ with a mean value of $10.4 \pm 2.3 \mathrm{~m} / \mathrm{s}$ and also a large range of BMI: from 15 to $81 \mathrm{~kg} / \mathrm{m}^{2}$ (mean value: $30 \pm 7 \mathrm{~kg} / \mathrm{m}^{2}$ ) with $22 \%$ normal, $39 \%$ overweight, $18 \%$ obese, $12 \%$ severely obese and $9 \%$ morbidly obese patients.

Patients with confirmed OSA were older and had higher BP (table 2).

A two-step meta-analysis was also performed. Results are provided in the online supplement. A limited heterogeneity was observed across the nine studies (online supplementary figure S1).

The univariate analysis for the one-step IPD meta-analysis (figure 2) showed that PWV was strongly related to age $(p<0.01)$, male gender $(p<0.01)$, SBP $(p<0.01)$, presence of diabetes $(p<0.01)$, presence of dyslipidaemia $(p=0.03)$ and ESS score $\geq 9(p=0.04)$, and was not related to severe OSA defined by AHI $\geq 30$ events/hour $(\mathrm{p}=0.14)$.

In multivariable analysis, PWV was independently associated with age, SBP and diabetes (all $\mathrm{p}<0.01$ ), whereas severe OSA was not significantly associated with PWV (figure 3).

\section{DISCUSSION}

To our knowledge, this is the first meta-analysis using individual patient data aiming to disentangle the respective influences of OSA and comorbidities on arterial stiffness. Most reviews and 
Estimate $(95 \% \mathrm{Cl})$, pvalue

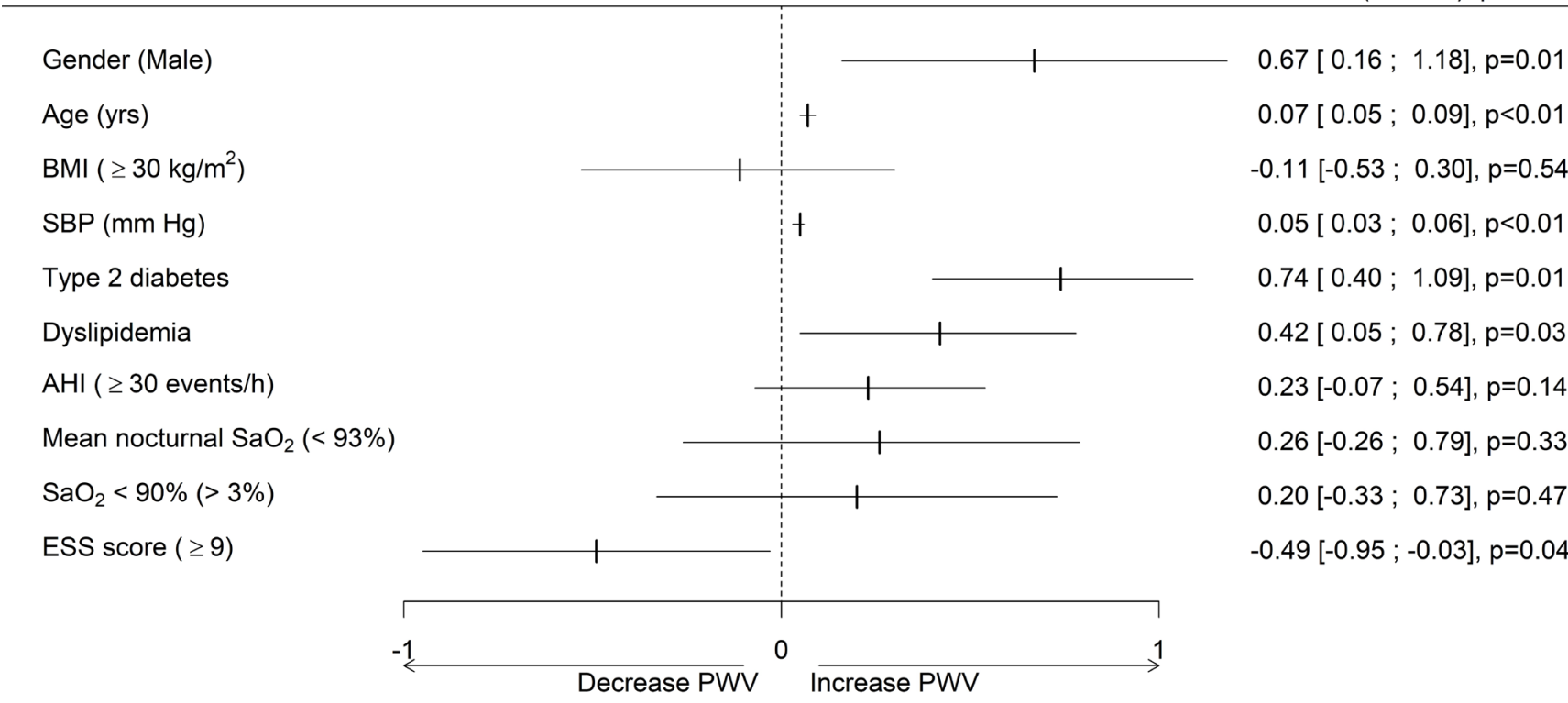

Figure 2 Forest plot of the factors associated with PWV (univariate analysis). AHI, Apnoea-Hypopnoea Index; BMI, Body Mass Index; ESS, Epworth Sleepiness Scale; PWV, pulse wave velocity; $\mathrm{SaO}_{2}$, oxygen saturation; SBP, systolic blood pressure.

previous meta-analyses used data from different groups with several different methods of arterial stiffness assessment. ${ }^{9}{ }^{10} \mathrm{~A}$ strength of our meta-analysis using individual patient data was that all data came from a single centre with standard operating procedures and long expertise in performing PWV and sleep studies. The use of complete individual datasets allowed subgroup analyses to be made, to adjust for covariates and hence increase power, as recommended by Bratton et al. ${ }^{31}$
The systematic review of Doonan et al, ${ }^{9}$ published in 2011, found 24 studies investigating the effect of OSA on arterial stiffness, while that of Phillips et $a l^{10}$ published 2 years later included 28 studies. A broad variety of techniques had been used to assess arterial stiffness, some studies used carotid-femoral PWV, other studies used brachial-ankle PWV and some authors only estimated arterial stiffness indirectly by derivatives such as the Augmentation Index. Although most studies found a significant

\section{Estimate $(95 \% \mathrm{Cl})$, pvalue}

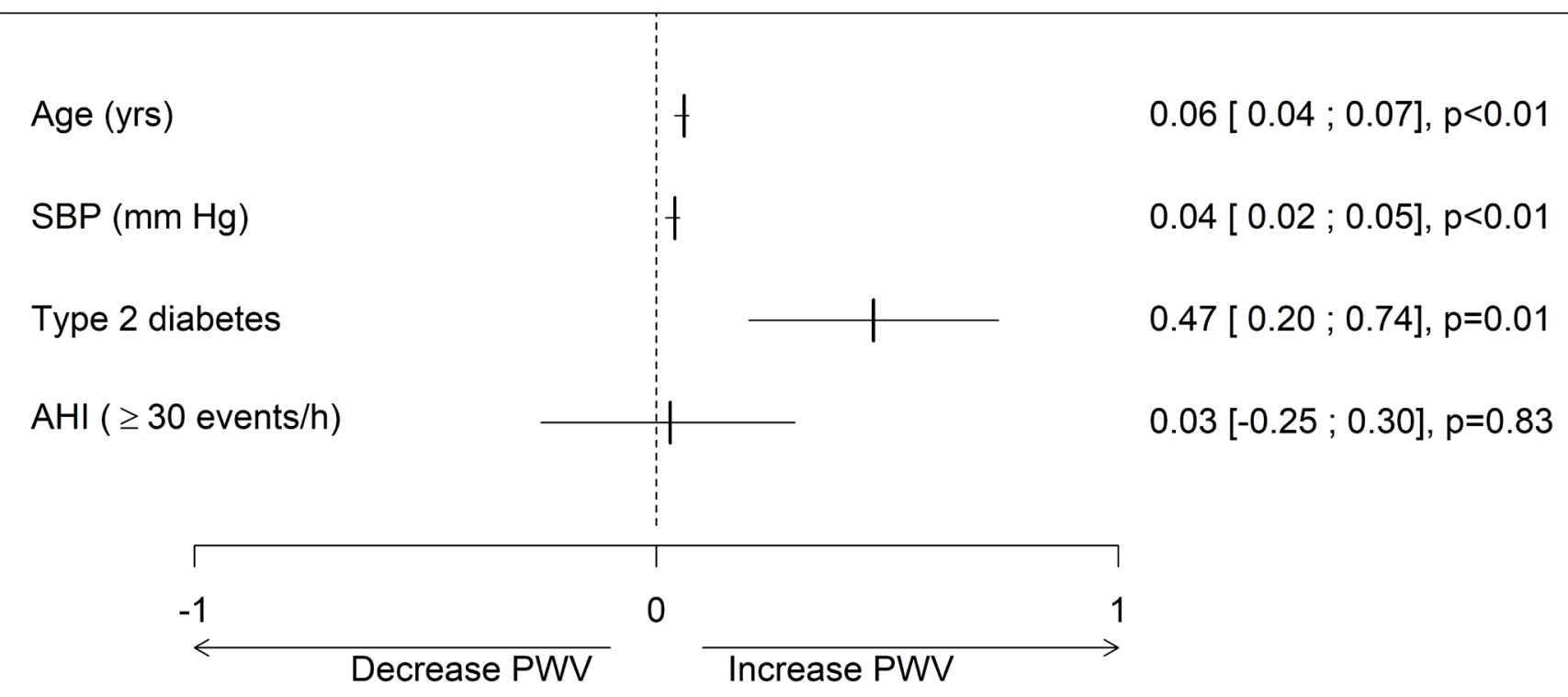

Figure 3 Forest plot of the independent factors associated with PWV (multivariate analysis). AHI, Apnoea-Hypopnoea Index; PWV, pulse wave velocity; SBP, systolic blood pressure. 
difference in arterial stiffness between controls and patients with OSA, almost a third did not find a difference. ${ }^{1419}$ Recently, Kim et al reported no association between OSA severity and arterial stiffness assessed by brachial-ankle PWV in a population of patients over 60 years of age. ${ }^{20}$ Ankle-brachial PWV, as opposed to carotid-femoral PWV used in our meta-analysis, is not the internationally recognised standard method of measuring arterial stiffness. Indeed, ankle-brachial PWV assesses arterial stiffness over the aortic segment and also in the lower limbs. The brachial-ankle PWV or the Cardio-Ankle Vascular Index (CAVI), another non-standard measure of arterial stiffness, should not be used in patients with peripheral arterial disease (PAD), a very highly prevalent condition in patients with OSA. ${ }^{32}$ Alberto et al carefully excluded patients with PAD in their study and only observed a non-significant trend between CAVI and nocturnal intermittent hypoxia. ${ }^{33}$

In the present study, we systematically used the gold standard carotid-femoral PWV methodology to assess arterial stiffness. In univariate analysis, ESS score $\geq 9$ was the only OSA parameter statistically associated with PWV. When studying non-selected OSA groups reflecting the diverse populations referred to a sleep clinic, the relationship between OSA and arterial stiffness is modest and only identifiable in univariate analysis in the patients with most severe OSA, who also have the highest prevalence of comorbidities.

As confirmed by our data, PWV and OSA syndrome share confounders. Besides age, BP is the strongest confounding factor of PWV. ${ }^{5}$ The prevalence of hypertension among patients with OSA is particularly high ${ }^{22}$ and OSA and hypertension are also linked in a dose-response fashion. ${ }^{3435}$

The association between age SBP and PWV is complex, as shown by AlGhatrif et al. ${ }^{36}$ In our study, the interaction between age and SBP was tested in the model and was found to be not significant $(p=0.06)$ showing that the impact of OSA on PWV remained unchanged. Obesity is very often associated with OSA syndrome. ${ }^{24}$ Seetho et al observed that normotensive obese subjects with OSA had a higher Augmentation Index than normotensive obese subjects without OSA. ${ }^{23}$ The Augmentation Index is sometimes used as a surrogate measure of arterial stiffness; however, it is highly dependent on vascular tone and cardiac characteristics. ${ }^{37}$ In obesity and metabolic syndrome, vascular tone is modified. In our analysis, confirming previous results in patients without OSA, ${ }^{7}$ obesity did not play a significant role in the increased arterial stiffness. As stated by Drager et al, while OSA is one of the consequences of obesity, it might not have a large additional influence on cardiovascular risk. ${ }^{38}$ Chirinos et al showed that CPAP treatment has an incremental effect over weight loss alone in reducing blood pressure, but the impact of combined therapy on arterial stiffness was not evaluated. ${ }^{24}$

Despite the use of brachial-ankle PWV, it is interesting to note that Kim et al observed a relationship between arterial stiffness and OSA severity in a subgroup of patients over the age of 60 with no other comorbidity or medical treatment. ${ }^{20}$ They hypothesised that chronic diseases (hypertension, diabetes and dyslipidaemia) increased arterial stiffness while the use of antihypertensive, antidiabetic or lipid-lowering drugs might have a positive impact on PWV, blunting the association between arterial stiffness and OSA severity. ${ }^{20}$ In a meta-analysis, Wang et al looked at studies only comparing controls to patients with OSA free of other cardiovascular risk factors and also concluded that carotidfemoral PWV is elevated by OSA per se. ${ }^{17}$ Our meta-analysis showed that arterial stiffness measured by PWV was marginally influenced by OSA in the presence of comorbidities. As stronger association might be found in the conduit arteries in of younger patients with OSA, which are more sensitive to sympathetic activation compared with the aorta. This requires further studies. The impact of OSA syndrome per se on the arteries seems negligible compared with the impact of hypertension, diabetes and dyslipidaemia. This might explain why CPAP treatment has relatively modest impact on BP or PWV. ${ }^{25} 3539$ Only patients with the most severe OSA with higher baseline values significantly benefit from CPAP in terms of BP reduction. ${ }^{40}{ }^{41}$ Careful management of OSA-associated comorbidities in combination with CPAP is more likely to have a greater impact on the occurrence of late cardiovascular events and survival.

\section{Limitations}

Data were merged from observational and interventional clinical trials. Therefore, the population might not be completely homogeneous depending on the specific inclusion and exclusion criteria of each trial. Moreover, while polygraphy was used in some studies, PSG was used in others, which might also have introduced some heterogeneity. Nevertheless, compared with other meta-analyses that included data from different centres with different techniques for PWV measurements, the impact was certainly small. Moreover, significant hypoxia was present in the control non-OSA group and was explained by documented COPD or morbid obesity and smoking, which were not included in the analysis. We cannot exclude a bias in patient recruitment, and our results might not be generalisable to the general OSA population. However, our population exhibited multiple comorbidities, which is clinically relevant. We observed a tendency to higher PWV in patients with OSA than in controls, which was not significant after adjustment in multivariable analysis, potentially owing to the small size of the control group. Finally, the cross-sectional nature of our meta-analysis does not allow us to explain any causality other than associations between the observed parameters.

\section{CONCLUSION}

Our individual patient meta-analysis showed that elevated arterial stiffness in patients with OSA is mainly driven by the conventional cardiovascular risk factors; age, BP and the presence of diabetes and apnoea severity indices had limited influence.

\section{Prior abstract publication/presentation}

- American Thoracic Society congress, 19-24 May 2016, San Francisco, CA, USA.

- European Society of Hypertension congress (26th European Meeting on Hypertension and Cardiovascular Protection), 10-13 June 2016, Paris, France.

Acknowledgements The authors are grateful to Marion Perrin for participating in statistical analyses (INSERM U1042, HP2 Laboratory, Grenoble Alpes University Hospital, France). We thank Alison Foote (Grenoble Alpes University Hospital, France) for critically editing the manuscript.

Contributors MJ-F, SB, RT, J-CB, SM, JLP: researched data, contributed to discussion, wrote the manuscript, reviewed/edited the manuscript. L-MG, MD: researched data, contributed to discussion, reviewed/edited the manuscript. JLP takes responsibility for the integrity of the work as a whole, including the data and analysis, from inception to revised article.

Funding This study received unrestricted grants from the endowment fund 'Agir pour les maladies chroniques'. This work was also supported by the French National Research Agency in the framework of the 'Investissements d'avenir' programme (ANR-15-IDEX-02).

Competing interests None of the authors has a competing interest with respect to this study except SM. SM works as a freelance specialist on pulse wave analysis and receives revenues from several medical device companies including Alam Medical whose device was used in this study. 
Patient consent Not required.

Ethics approval Comité de Protection des Personnes, Sud Est V IRB0006705 France.

Provenance and peer review Not commissioned; externally peer reviewed.

\section{REFERENCES}

1 Laurent S, Cockcroft J, Van Bortel L, et al. Expert consensus document on arterial stiffness: methodological issues and clinical applications. Eur Heart $J$ 2006;27:2588-605.

2 Mancia G, Fagard R, Narkiewicz K, et al. 2013 ESH/ESC guidelines for the management of arterial hypertension: the task force for the management of arterial hypertension of the European Society of Hypertension (ESH) and of the European Society of Cardiology (ESC). Eur Heart J 2013;34:2159-219.

3 Ben-Shlomo Y, Spears M, Boustred C, et al. Aortic pulse wave velocity improves cardiovascular event prediction: an individual participant meta-analysis of prospective observational data from 17,635 subjects. J Am Coll Cardiol 2014;63:636-46.

4 Vlachopoulos C, Aznaouridis K, Stefanadis C. Aortic stiffness for cardiovascular risk prediction: just measure it, just do it!. J Am Coll Cardiol 2014;63:647-9.

5 Cecelja M, Chowienczyk P. Dissociation of aortic pulse wave velocity with risk factors for cardiovascular disease other than hypertension: a systematic review. Hypertension 2009:54:1328-36.

6 Vlachopoulos C, Aznaouridis K, Stefanadis C. Prediction of cardiovascular events and all-cause mortality with arterial stiffness: a systematic review and meta-analysis. J Am Coll Cardiol 2010;55:1318-27.

7 Desamericq G, Tissot CM, Akakpo S, et al. Carotid-femoral pulse wave velocity is not increased in obesity. Am J Hypertens 2015;28:546-51.

8 Lévy P, Kohler M, McNicholas WT, et al. Obstructive sleep apnoea syndrome. Nat Rev Dis Primers 2015;1:15015.

9 Doonan RJ, Scheffler P, Lalli M, et al. Increased arterial stiffness in obstructive sleep apnea: a systematic review. Hypertens Res 2011;34:23-32.

10 Phillips CL, Butlin M, Wong KK, et al. Is obstructive sleep apnoea causally related to arterial stiffness? A critical review of the experimental evidence. Sleep Med Rev 2013;17:7-18.

11 Drager LF, Bortolotto LA, Lorenzi MC, et al. Early signs of atherosclerosis in obstructive sleep apnea. Am J Respir Crit Care Med 2005;172:613-8.

12 Phillips C, Hedner J, Berend N, et al. Diurnal and obstructive sleep apnea influences on arterial stiffness and central blood pressure in men. Sleep 2005;28:604-9.

13 Tsioufis C, Thomopoulos K, Dimitriadis K, et al. The incremental effect of obstructive sleep apnoea syndrome on arterial stiffness in newly diagnosed essential hypertensive subjects. J Hypertens 2007;25:141-6.

14 Protogerou AD, Laaban JP, Czernichow S, et al. Structural and functional arterial properties in patients with obstructive sleep apnoea syndrome and cardiovascular comorbidities. J Hum Hypertens 2008;22:415-22.

15 Suh BC, Chung PW, Moon HS, et al. Association between pulse wave velocity and nerve conduction study in diabetic patients. Eur Neurol 2010;64:219-23.

16 Chen CY, Chen CL, Yu CC. Obstructive sleep apnea is independently associated with arterial stiffness in ischemic stroke patients. J Neurol 2015;262:1247-54.

17 Wang J, Yu W, Gao M, et al. Impact of obstructive sleep apnea syndrome on endothelial function, arterial stiffening, and serum inflammatory markers: an updated meta-analysis and metaregression of 18 studies. J Am Heart Assoc 2015;4:e002454.

18 Chung S, Yoon IY, Lee $\mathrm{CH}_{\text {, et }}$ al. The association of nocturnal hypoxemia with arterial stiffness and endothelial dysfunction in male patients with obstructive sleep apnea syndrome. Respiration 2010;79:363-9.

19 Baguet JP, Nadra M, Barone-Rochette G, et al. Early cardiovascular abnormalities in newly diagnosed obstructive sleep apnea. Vasc Health Risk Manag 2009;5:1063-73.
20 Kim T, Lee CS, Lee SD, et al. Impacts of comorbidities on the association between arterial stiffness and obstructive sleep apnea in the elderly. Respiration 2015;89:304-11.

21 Bailly S, Destors M, Grillet Y, et al. Obstructive sleep apnea: a cluster analysis at time of diagnosis. PLoS One 2016;11:e0157318.

22 Baguet JP, Lévy P, Barone-Rochette G, et al. Masked hypertension in obstructive sleep apnea syndrome. J Hypertens 2008;26:885-92.

23 Seetho IW, Parker RJ, Craig S, et al. Obstructive sleep apnea is associated with increased arterial stiffness in severe obesity. J Sleep Res 2014;23:700-8.

24 Chirinos JA, Gurubhagavatula I, Teff K, et al. CPAP, weight loss, or both for obstructive sleep apnea. N Engl J Med 2014;370:2265-75.

25 Vlachantoni IT, Dikaiakou E, Antonopoulos CN, et al. Effects of continuous positive airway pressure (CPAP) treatment for obstructive sleep apnea in arterial stiffness: a meta-analysis. Sleep Med Rev 2013;17:19-28.

26 Borel JC, Tamisier R, Gonzalez-Bermejo J, et al. Noninvasive ventilation in mild obesity hypoventilation syndrome: a randomized controlled trial. Chest 2012;141:692-702.

27 Berry RB, Budhiraja R, Gottlieb DJ, et al. Rules for scoring respiratory events in sleep: update of the 2007 AASM Manual for the Scoring of Sleep and Associated Events. Deliberations of the Sleep Apnea Definitions Task Force of the American Academy of Sleep Medicine. J Clin Sleep Med 2012;8:597-619.

28 Asmar R, Benetos A, Topouchian J, et al. Assessment of arterial distensibility by automatic pulse wave velocity measurement. Validation and clinical application studies. Hypertension 1995;26:485-90.

29 Mathieu S, Pereira B, Couderc M, et al. No significant changes in arterial stiffness in patients with ankylosing spondylitis after tumour necrosis factor alpha blockade treatment for 6 and 12 months. Rheumatology 2013;52:204-9.

30 Van Bortel LM, Laurent S, Boutouyrie P, et al. Expert consensus document on the measurement of aortic stiffness in daily practice using carotid-femoral pulse wave velocity. J Hypertens 2012;30:445-8.

31 Bratton DJ, Stradling JR, Barbé F, et al. Effect of CPAP on blood pressure in patients with minimally symptomatic obstructive sleep apnoea: a meta-analysis using individual patient data from four randomised controlled trials. Thorax 2014:69:1128-35.

32 Schaefer CA, Adam L, Weisser-Thomas J, et al. High prevalence of peripheral arterial disease in patients with obstructive sleep apnoea. Clin Res Cardiol 2015;104:719-26.

33 Alberto EC, Tanigawa T, Maruyama K, et al. Relationships between nocturnal intermittent hypoxia, arterial stiffness and cardiovascular risk factors in a communitybased population: the Toon health study. J Atheroscler Thromb 2014;21:1290-7.

34 Peppard PE, Young T, Palta M, et al. Prospective study of the association between sleep-disordered breathing and hypertension. N Engl J Med 2000;342:1378-84.

35 Pepin JL, Borel AL, Tamisier R, et al. Hypertension and sleep: overview of a tight relationship. Sleep Med Rev 2014;18:509-19.

36 AlGhatrif M, Strait JB, Morrell CH, et al. Longitudinal trajectories of arterial stiffness and the role of blood pressure: the Baltimore Longitudinal Study of Aging. Hypertension 2013;62:934-41

37 Kelly RP, Millasseau SC, Ritter JM, et al. Vasoactive drugs influence aortic augmentation index independently of pulse-wave velocity in healthy men. Hypertension 2001;37:1429-33.

38 Drager LF, Togeiro SM, Polotsky VY, et al. Obstructive sleep apnea: a cardiometabolic risk in obesity and the metabolic syndrome. J Am Coll Cardiol 2013;62:569-76.

39 Kartali N, Daskalopoulou E, Geleris P, et al. The effect of continuous positive airway pressure therapy on blood pressure and arterial stiffness in hypertensive patients with obstructive sleep apnea. Sleep and Breathing 2014;18:635-40.

40 Bratton DJ, Gaisl T, Wons AM, et al. CPAP vs Mandibular advancement devices and blood pressure in patients with obstructive sleep apnea: a systematic review and meta-analysis. JAMA 2015;314:2280-93.

41 Martínez-García MA, Capote F, Campos-Rodríguez F, et al. Effect of CPAP on blood pressure in patients with obstructive sleep apnea and resistant hypertension: the HIPARCO randomized clinical trial. JAMA 2013:310:2407-15. 